International Journal of Pure and Applied Mathematics

Volume 89 No. 1 2013, 35-39

ISSN: 1311-8080 (printed version); ISSN: 1314-3395 (on-line version)

url: http://www.ijpam.eu

doi: http://dx.doi.org/10.12732/ijpam.v89i1.5

ijpam.eu

\title{
POISSON APPROXIMATION FOR RANDOM SUMS OF GEOMETRIC RANDOM VARIABLES
}

\author{
K. Teerapabolarn \\ Department of Mathematics \\ Faculty of Science \\ Burapha University \\ Chonburi, 20131, THAILAND
}

\begin{abstract}
In this paper, we determine bounds with different Poisson mean for the total variation distance between the distribution of random sums of independent geometric random variables and an appropriate Poisson distribution. Two examples have been given to illustrate the results obtained.
\end{abstract}

AMS Subject Classification: 62E17, 60F05, 60G05

Key Words: geometric random variable, Poisson approximation, random sums, total variation distance

\section{Introduction}

Let $X_{1}, \ldots, X_{n}$ be $n(n \in \mathbb{N})$ independently distributed geometric random variables, each with $P\left(X_{i}=k\right)=\left(1-p_{i}\right)^{k} p_{i}, k=0,1, \ldots$, and let $S_{n}=\sum_{i=1}^{n} X_{i}$ and $U_{\lambda}$ a Poisson random variable with mean $\lambda$. It is well-known that if all $q_{i}=1-p_{i}$ are small, then the distribution of $S_{n}$, denoted by $\mathcal{L}\left(S_{n}\right)$, can be approximated by an appropriate Poisson distribution with mean $\lambda$, denoted by $\mathcal{L}\left(U_{\lambda}\right)$. In this case, Teerapabolarn and Wongkasem [2] gave a bound for the total variation distance between the distributions of $S_{n}$ and $U_{\lambda_{n}}$ as follows:

Received: May 22, 2013

(c) 2013 Academic Publications, Ltd. url: www.acadpubl.eu 


$$
\begin{aligned}
d\left(\mathcal{L}\left(S_{n}\right), \mathcal{L}\left(U_{\lambda_{n}}\right)\right) & =\sup _{A \subseteq \mathbb{N} \cup\{0\}}\left|P\left(S_{n} \in A\right)-P\left(U_{\lambda_{n}} \in A\right)\right| \\
& \leq \sum_{i=1}^{n} \min \left\{\frac{1-e^{-\lambda_{n}}}{\lambda_{n} p_{i}}, 1\right\} \frac{q_{i}^{2}}{p_{i}}
\end{aligned}
$$

for $\lambda_{n}=\sum_{i=1}^{n} \frac{q_{i}}{p_{i}}$. For $\lambda_{n}=\sum_{i=1}^{n} q_{i}$, Vellaisamy and Upadhye [3] gave a bound in the form of

$$
d\left(\mathcal{L}\left(S_{n}\right), \mathcal{L}\left(U_{\lambda_{n}}\right)\right) \leq \sum_{i=1}^{n} \min \left\{\frac{0.42888}{\sqrt{\lambda_{n}}}, 1\right\} \frac{q_{i}^{2}}{p_{i}}
$$

Let us consider the sum $S_{N}=\sum_{i=1}^{N} X_{i}$, where $N$ is a non-negative integer valued random variable and independent of the $X_{i}$ 's. The sum is called random sums of independent geometric random random variables. In this study, we are interest to approximate $\mathcal{L}\left(S_{N}\right)$ by $\mathcal{L}\left(U_{\lambda}\right)$ when $\lambda=E\left(\lambda_{N}\right)$, which are in Section 2. We give some examples to illustrate the results of this study in the last section.

\section{Result}

The following theorem presents bounds with different Poisson mean for the total variation distance between $\mathcal{L}\left(S_{N}\right)$ and $\mathcal{L}\left(U_{\lambda}\right)$.

Theorem 2.1. With the above definitions:

1. For $\lambda_{N}=\sum_{i=1}^{N} \frac{q_{i}}{p_{i}}$,

$$
\begin{aligned}
d\left(\mathcal{L}\left(S_{N}\right), \mathcal{L}\left(U_{\lambda}\right)\right) \leq & \min \left\{1, \sqrt{\frac{2}{e \lambda}}\right\} E\left|\lambda_{N}-\lambda\right| \\
& +\min \left\{E\left(\frac{1-e^{-\lambda_{N}}}{\lambda_{N}} \sum_{i=1}^{N} \frac{q_{i}^{2}}{p_{i}^{2}}\right), E\left(\sum_{i=1}^{N} \frac{q_{i}^{2}}{p_{i}}\right)\right\} .
\end{aligned}
$$

2. For $\lambda_{N}=\sum_{i=1}^{N} q_{i}$,

$$
\begin{aligned}
d\left(\mathcal{L}\left(S_{N}\right), \mathcal{L}\left(U_{\lambda}\right)\right) \leq & \min \left\{1, \sqrt{\frac{2}{e \lambda}}\right\} E\left|\lambda_{N}-\lambda\right| \\
& +\min \left\{E\left(\frac{0.42888}{\sqrt{\lambda_{N}}} \sum_{i=1}^{N} \frac{q_{i}^{2}}{p_{i}}\right), E\left(\sum_{i=1}^{N} \frac{q_{i}^{2}}{p_{i}}\right)\right\} .
\end{aligned}
$$


Proof. 1. We have

$$
\begin{aligned}
& d\left(\mathcal{L}\left(S_{N}\right), \mathcal{L}\left(U_{\lambda}\right)\right) \leq d\left(\mathcal{L}\left(S_{N}\right), \mathcal{L}\left(U_{\lambda_{N}}\right)\right)+d\left(\mathcal{L}\left(U_{\lambda_{N}}\right), \mathcal{L}\left(U_{\lambda}\right)\right) \\
&=\sum_{n=0}^{\infty} P(N=n) d\left(\mathcal{L}\left(S_{n}\right), \mathcal{L}\left(U_{\lambda_{n}}\right)\right)+d\left(\mathcal{L}\left(U_{\lambda_{N}}\right), \mathcal{L}\left(U_{\lambda}\right)\right) \\
& \leq \sum_{n=0}^{\infty} P(N=n) \sum_{i=1}^{n} \min \left\{\frac{1-e^{-\lambda}}{\lambda p_{i}}, 1\right\} \frac{q_{i}^{2}}{p_{i}} \\
& \quad+\min \left\{1, \sqrt{\left.\frac{2}{e \lambda}\right\} E\left|\lambda_{N}-\lambda\right|}\right. \\
&=\min \left\{1, \sqrt{\frac{2}{e \lambda}}\right\} E\left|\lambda_{N}-\lambda\right| \\
& \quad+\min \left\{E\left(\frac{1-e^{-\lambda_{N}}}{\lambda_{N}} \sum_{i=1}^{N} \frac{q_{i}^{2}}{p_{i}^{2}}\right), E\left(\sum_{i=1}^{N} \frac{q_{i}^{2}}{p_{i}}\right)\right\}
\end{aligned}
$$

where the first and second terms of the right hand side of (2.3) follow (1.1) and [1] on pp. 12, respectively.

2. Substituting the bound in the first term of the right hand side of (2.3) by the bound in (1.2), we have

$$
\begin{aligned}
d\left(\mathcal{L}\left(S_{N}\right), \mathcal{L}\left(U_{\lambda}\right)\right) \leq \sum_{n=0}^{\infty} & P(N=n) \sum_{i=1}^{n} \min \left\{\frac{0.42888}{\sqrt{\lambda_{n}}}, 1\right\} \frac{q_{i}^{2}}{p_{i}} \\
& +\min \left\{1, \sqrt{\left.\frac{2}{e \lambda}\right\} E\left|\lambda_{N}-\lambda\right|}\right. \\
=\min & \left\{1, \sqrt{\left.\frac{2}{e \lambda}\right\} E\left|\lambda_{N}-\lambda\right|}\right. \\
& +\min \left\{E\left(\frac{0.42888}{\sqrt{\lambda_{N}}} \sum_{i=1}^{N} \frac{q_{i}^{2}}{p_{i}}\right), E\left(\sum_{i=1}^{N} \frac{q_{i}^{2}}{p_{i}}\right)\right\} .
\end{aligned}
$$

Hence, the proof is complete.

If $X_{i}$ 's are identically distributed, then the following corollary is an immediately consequence of the Theorem 2.1,

Corollary 2.1. If $p_{1}=p_{2}=\cdots=p$, then we have the following: 
1. For $\lambda_{N}=\frac{N q}{p}$,

$$
\begin{aligned}
d\left(\mathcal{L}\left(S_{N}\right), \mathcal{L}\left(U_{\lambda}\right)\right) \leq \min & \left\{1, \sqrt{\frac{2 p}{e E(N) q}}\right\} \frac{q}{p} E|N-E(N)| \\
& +\min \left\{E\left(1-e^{-\frac{N q}{p}}\right) \frac{q}{p}, E(N) \frac{q^{2}}{p}\right\}
\end{aligned}
$$

2. For $\lambda_{N}=N q$,

$$
\begin{aligned}
d\left(\mathcal{L}\left(S_{N}\right), \mathcal{L}\left(U_{\lambda}\right)\right) \leq \min & \left\{1, \sqrt{\frac{2}{e E(N) q}}\right\} q E|N-E(N)| \\
& +\min \left\{\frac{0.42888 q^{\frac{3}{2}} E(\sqrt{N})}{p}, E(N) \frac{q^{2}}{p}\right\} .
\end{aligned}
$$

\section{Examples}

This section, we give two examples to illustrate the results in the case of $X_{i}$ 's are identically distributed, which are in the Corollary 2.1.

Example 3.1. For $n(n \in \mathbb{N})$ is fixed, let $N$ be a positive integer-valued random variable with probability function

$$
P(N=k)= \begin{cases}\frac{1}{2}, & k=n \\ \frac{1}{2}, & k=2 n \\ 0, & \text { otherwise. }\end{cases}
$$

Therefore $E(N)=\frac{3 n}{2}$ and $E|N-E(N)|=\frac{n}{2}$. Let $p_{1}=p_{2}=\cdots=p$, then we have

$$
d\left(\mathcal{L}\left(S_{N}\right), \mathcal{L}\left(U_{\frac{3 n q}{2 p}}\right)\right) \leq \min \left\{1, \sqrt{\frac{4 p}{3 e n q}}\right\} \frac{n q}{2 p}+\min \left\{\frac{q}{p}, \frac{3 n q^{2}}{2 p}\right\}
$$

and

$$
d\left(\mathcal{L}\left(S_{N}\right), \mathcal{L}\left(U_{\frac{3 n q}{2}}\right)\right) \leq \min \left\{1, \sqrt{\frac{4}{3 e n q}}\right\} \frac{n q}{2}+\min \left\{0.42888 \sqrt{\frac{3 n q^{3}}{2 p^{2}}}, \frac{3 n q^{2}}{2 p}\right\}
$$


Example 3.2. Let $N$ be a positive integer-valued random variable with probability function

$$
P(N=n)=\frac{1}{2^{n}}, n=1,2, \ldots,
$$

then we have $E(N)=2$ and $E|N-E(N)|=1$. If $p_{1}=p_{2}=\cdots=p$, then we obtain

$$
d\left(\mathcal{L}\left(S_{N}\right), \mathcal{L}\left(U_{\frac{2 q}{p}}\right)\right) \leq \min \left\{1, \sqrt{\frac{p}{e q}}\right\} \frac{q}{p}+\min \left\{\frac{q}{p}, \frac{2 q^{2}}{p}\right\}
$$

and

$$
d\left(\mathcal{L}\left(S_{N}\right), \mathcal{L}\left(U_{2 q}\right)\right) \leq \min \left\{1, \sqrt{\frac{1}{e q}}\right\} q+\min \left\{0.42888 \sqrt{\frac{2 q^{3}}{p^{2}}}, \frac{2 q^{2}}{p}\right\} .
$$

\section{References}

[1] A.D. Barbour, L. Holst, S. Janson, Poisson Approximation, Oxford Studies in Probability, 2, Clarendon Press, Oxford (1992).

[2] K. Teerapabolarn, P. Wongkasem, Poisson approximation for independent geometric random variables, Int. Math. Forum, 2 (2007), 3211-3218.

[3] P. Vellaisamy, N.S. Upadhye, Compound negative binomial approximations for sums of random variables, Probab. Math. Statist., 29 (2009), 205-226. 
